\title{
Immunotherapy for Bladder Cancer: Changing the Landscape
}

\author{
Laeeq Malik \\ ${ }^{1}$ Institute for Drug Development, Cancer Therapy and Research Center, University of Texas Health Science \\ Center at San Antonio, San Antonio, TX, USA \\ Correspondence: Laeeq Malik, MD, FRACP, Institute for Drug Development, Cancer Therapy and Research \\ Center, University of Texas Health Science Center at San Antonio, San Antonio, TX, USA. Tel: 1-210-450-5947. \\ E-mail: Malikl@uthscsa.edu
}

\author{
Received: August 21, 2014 Accepted: September 9, 2014 Online Published: September 25, 2014 \\ doi:10.5539/cco.v3n2p36 URL: http://dx.doi.org/10.5539/cco.v3n2p36
}

\begin{abstract}
Systemic chemotherapy plays a central role in the management of metastatic bladder cancer. Although responses may be observed initially, overall survival with multiagent chemotherapy is still limited. No significant improvements have been made by novel cytotoxic or targeted agents in last decade. There exists an urgent need for the development of novel therapeutic agents to improve outcomes for these patients. In this paper, we review the role of immunotherapy, the programmed death 1 (PD-1) receptor and its ligands (PD-L1/2), and ongoing drug development efforts to block this pathway in bladder cancer, focusing on the currently available data from ongoing clinical trials.
\end{abstract}

Keywords: bladder cancer, immunotherapy, PD-1

\section{Introduction}

Bladder cancer is the most common malignant disease involving the urinary system. Approximately 74,690 patients will be diagnosed with bladder cancer in 2014 ("SEER Stat Fact Sheets: Bladder Cancer", 2014). About one third of bladder cancer patients develop locally invasive or metastatic disease (Raghavan, 2003). Systemic chemotherapy is the standard approach for patients with inoperable locally advanced or metastatic bladder cancer. Although responses may be observed initially, the median survival with multiagent chemotherapy is about 13 months (Loehrer et al., 1992). In Europe, vinflunine is approved as a treatment option for patients with advanced urothelial cancer who have failed a prior platinum-containing regimen (Bellmunt et al., 2009). To date, in the USA, there is no Food and Drug Administration (FDA) approved second-line chemotherapy for patients with metastatic bladder cancer and guidelines continue to emphasize patient enrollment into a clinical trial. Active single agents not incorporated in the initial regimen may have activity in some patients.

There had been a plateau in the development of new effective agents for this aggressive malignancy. While there is an increasing understanding of the molecular biology and signaling pathways underlying bladder cancer development and progression, no targeted agents currently have proven a significant role in the treatment of urothelial bladder cancer. There is increasing evidence that some tumors can evade adaptive immunity and disrupt T-cell checkpoint pathways. More recently, efforts have targeted and activated the immune system to treat various malignancies. It is imperative to develop more effective, less toxic agents by incorporating these novel findings to improve outcomes in bladder cancer.

In this paper, we review the role of immunotherapy, the programmed death 1 (PD-1) receptor and its ligands (PD-L1/2), and ongoing drug development efforts to block this pathway in bladder cancer, focusing on the currently available data from ongoing clinical trials.

\section{Bacille Calmette-Guerin (BCG) Immunotherapy}

BCG, a live attenuated strain of Mycobacterium bovis, was initially used in man as a vaccine against tuberculosis in 1921. Its first use in cancer medicine was reported in a case series of nine patients in 1976 (Morales, Eidinger, \& Bruce, 1976). It has now become a mainstay of adjunctive therapy for superficial bladder cancer. Although, other intravesical agents have been evaluated in superficial bladder cancer, but none has proven to be more effective than BCG. Its mechanism of action in the treatment of bladder cancer is still not fully known and is beyond the scope of this review. Exposure to BCG suppresses tumor cell growth in a dose-dependent manner, and has an antiproliferative effect on certain tumor cell lines (Rajala et al., 1992). Exposure to BCG triggers a 
local immune response as evidenced by infiltration of bladder with inflammatory cells (Mitropoulos, 2005). A significant urinary secretion of several cytokines can be detected within 24 hours of BCG instillation, with a maximum titer after 2-8 hours and a decrease to normal values within 24 hours (Taniguchi et al., 1999). This sharp rise in urine level of cytokines after BCG instillation is suggestive of the immunological character of a BCG therapy (Elsasser-Beile et al., 2000). The presence of CD4+ helper, CD8+ cytolytic T cells and natural killer cells is necessary to induce antitumor effects (Ratliff, Gillen, \& Catalona, 1987). Once an effective TH1 cellular response has been generated, cellular activation leads to amplification of effector cells that are capable of eliminating bladder cancer cells and producing cytokines to regulate immune response.

The American Urological Association and the European Association of Urology bladder cancer guidelines recommend a course of intravesical BCG for patients with stage T1, Tis and high-grade Ta tumors (Babjuk et al., 2008; Hall et al., 2007). The short-term and long-term efficacy of BCG in the treatment of patients with superficial bladder cancer has been extensively studied. It has been shown to delay tumor progression (and a need for subsequent cystectomy) and improve overall survival (Herr, 1997). The survival rate at five years following BCG treatment is similar to that achieved after immediate cystectomy in these patients. In a systematic review of 585 patients with Ta or T1 disease that were involved in six randomized trials, BCG had significantly fewer recurrences at 12 months (Shelley et al., 2000). Intravesical BCG also significantly reduces the risk of long-term treatment failure. This was demonstrated in a meta-analysis of 700 patients with carcinoma in situ who were treated in nine randomized trials, $68 \%$ patients achieved a complete response with the BCG (Sylvester, van der Meijden, Witjes, \& Kurth, 2005). After a median followup of 3.6 years, 46.7\% patients with prior BCG had no evidence of disease.

Unfortunately, nearly $40 \%$ of patients with superficial bladder cancer will fail BCG therapy (Witjes, 2006). There are many reasons cited for BCG failure including insufficient treatment, occult invasive or metastatic disease, inadequate immune response, gradual waning of the immune response or natural resistance associated macrophage protein 1 (NRAMP1) gene polymorphism (Zlotta, Fleshner, \& Jewett, 2009). By acknowledging the limitations of BCG and with the newer discoveries in human cancer immunology, significant efforts are currently being made to again transform the treatment of advanced bladder cancer.

\section{Targetting Cytotoxic T-lymphocyte Antigen 4 (CTLA-4)}

The BCG success story paves the way to further explore a potential role of immunotherapeutic agents for advanced bladder cancer. In the last decade, much has been learned about the immunobiology of various tumors. Most of the discoveries in human cancer immunology originate from studies of melanoma, a cancer shown to be among the most immunogenic of all tumors. The potential therapeutic role of immunotherapy in augmenting the antitumor immune responses against cancer continues to expand. We have learned that the activation of cellular immunity begins when $\mathrm{T}$ cells recognize peptide fragments of intracellular proteins that are expressed on the surface of antigen presenting cells bound to specific major histocompatibility complex molecules (L. Chen \& Flies, 2013). This interaction requires the presence of a costimulatory molecule (B7) and activation results in upregulation of CTLA-4. The CTLA-4 receptor on T lymphocytes is a negative regulator of T cell activation that outcompetes CD28 for binding to B7 on antigen presenting cells (Peggs, Quezada, Korman, \& Allison, 2006). CTLA-4 thereby serves as a physiologic "control" on the activated immune system.

Ipilimumab is a monoclonal antibody to CTLA-4 that can prevent this feedback inhibition, resulting in an immune response against the tumor. Treatment with ipilimumab significantly increases median overall survival in both previously untreated and treated patients with metastatic melanoma (Hodi et al., 2010). Immune monitoring studies of patients with bladder cancer who were treated with two cycles of preoperative ipilimumab, have shown that treatment with anti-CTLA-4 antibody leads to an increase in the population of CD4+ inducible costimulator cells (CD4+ICOShi) in both tumor tissues and peripheral blood (Carthon et al., 2010). The preliminary data also indicated that an increased frequency of CD4+ inducible costimulator cells correlated with increased likelihood of clinical benefit. Further work is needed to investigate its role as a potential biomarker for correlation with clinical outcome in patients with metastatic disease who receive treatment with anti-CTLA-4. Ipilimumab related adverse events observed in this study were mostly reversible and manageable. Ipilimumab is currently being evaluated in a Phase II study in combination with cisplatin and gemcitabine for advanced urothelial cancer ("Phase II Trial of Gemcitabine, Cisplatin, Plus Ipilimumab as First-line Treatment for Patients With Metastatic Urothelial Carcinoma: Hoosier Oncology Group GU10-148").

\section{PD-1 Pathway Inhibitors}

Encouraging clinical data have demonstrated that therapies focused on enhancing T-cell responses against cancer can result in a significant survival benefit in patients with advanced cancer (Chen, Irving, \& Hodi, 2012; Hodi et 
al., 2010). PD-1 is an inhibitory receptor expressed on T cells following T-cell activation, that is sustained in states of chronic stimulation such as in cancer (Blank \& Mackensen, 2007). PD-L1 is an extracellular protein that down regulates immune responses primarily in peripheral tissues through binding to its two receptors PD-1 and B7.1. Ligation of PD-L1 with PD-1 inhibits T-cell proliferation, cytokine production, and cytolytic activity, leading to the functional inactivation or exhaustion of $\mathrm{T}$ cells. B7.1 is a molecule expressed on antigen-presenting cells and activated T cells. PD-L1 binding to B7.1 on T cells and antigen-presenting cells can mediate downregulation of immune responses, including inhibition of T-cell activation and cytokine production (Butte, Keir, Phamduy, Sharpe, \& Freeman, 2007; Yang et al., 2011). PD-L1 expression is prevalent in many human tumors. Overexpression of PD-L1 on tumor cells has been reported to impede antitumor immunity, resulting in immune evasion (Blank \& Mackensen, 2007). Elevated PD-L1 expression on tumor cells is possibly associated with a poor prognosis in patients with bladder cancer (Mu, Huang, Chen, Chen, \& Zhang, 2011). Preclinical data has shown that interruption of the interaction between PD-L1 and PD-1 in mouse tumor models lead to antitumor effects (Iwai et al., 2002) (Strome et al., 2003). Therefore, interruption of the PD-L1/PD-1 pathway represents an attractive strategy to reinvigorate tumor-specific T-cell immunity. Table 1 lists ongoing clinical trials of anti-PD-1 drugs for urothelial and other solid tumors.

Table 1. Ongoing Clinical Trials of Anti-PD-1 drugs for urothelial and other solid tumors

\begin{tabular}{|c|c|c|c|}
\hline Compound & Clinical trial No & Indication & Phase \\
\hline $\begin{array}{l}\text { Ipilimumab in combination } \\
\text { with cisplatin and gemcitabine }\end{array}$ & NCT01524991 & Urothelial cancer & II \\
\hline MPDL3280A & NCT02108652 & Urothelial cancer & II \\
\hline $\begin{array}{l}\text { MPDL3280A, ipilimumab, } \\
\text { interferon alfa-2b }\end{array}$ & NCT02174172 & $\begin{array}{l}\text { Urothelial cancer } \\
\text { Advanced cancer }\end{array}$ & I \\
\hline MPDL3280A, bevacizumab & NCT01984242 & Renal cancer & II \\
\hline MPDL3280A, erlotinib & NCT02013219 & Non-small cell lung cancer & I \\
\hline MPDL3280A, vemurafenib & NCT01656642 & Malignant melanoma & I \\
\hline MPDL3280A, cobimetinib & NCT01988896 & Advanced cancer (including urothelial) & I \\
\hline MPDL3280A & NCT02008227 & Non-small cell lung cancer & III \\
\hline MEDI4736 & NCT01693562 & $\begin{array}{l}\text { Urothelial cancer } \\
\text { Advanced cancer }\end{array}$ & I \\
\hline $\begin{array}{l}\text { MEDI4736, dabrafenib } \\
\text { trametinib }\end{array}$ & NCT02027961 & Malignant melanoma & I \\
\hline MEDI4736 & NCT02087423 & Non-small cell lung cancer & II \\
\hline MEDI4736, gefitinib & NCT02088112 & Non-small cell lung cancer & I \\
\hline MEDI4736 & NCT02125461 & Non-small cell lung cancer & III \\
\hline MEDI4736, tremelimumab & NCT02000947 & Non-small cell lung cancer & I \\
\hline Lambrolizumab & NCT02054806 & Advanced cancer (including urothelial) & I \\
\hline Lambrolizumab & NCT01848834 & $\begin{array}{l}\text { Urothelial cancer } \\
\text { Breast cancer } \\
\text { Head and neck cancer Gastric cancer }\end{array}$ & I \\
\hline Lambrolizumab, PF-05082566 & NCT02179918 & Advanced cancer (including urothelial) & I \\
\hline Lambrolizumab & NCT02142738 & Non-small cell lung cancer & III \\
\hline Nivolumab, ipilimumab & NCT01844505 & Malignant melanoma & III \\
\hline $\begin{array}{l}\text { Nivolumab, ipilimumab, } \\
\text { bevacizumab }\end{array}$ & NCT02210117 & Renal cancer & II \\
\hline Nivolumab, ipilimumab & NCT01928394 & $\begin{array}{l}\text { Urothelial cancer } \\
\text { Breast cancer } \\
\text { Gastric cancer } \\
\text { Pancreatic cancer } \\
\text { Small cell lung cancer }\end{array}$ & $\mathrm{I} / \mathrm{II}$ \\
\hline Nivolumab, ipilimumab & NCT01927419 & Malignant melanoma & II \\
\hline $\begin{array}{l}\text { Nivolumab, ipilimumab, } \\
\text { bevacizumab }\end{array}$ & NCT02017717 & Glioblastoma & II \\
\hline Nivolumab & NCT02066636 & Non-small cell lung cancer & III \\
\hline Nivolumab & NCT01668784 & Renal cancer & III \\
\hline
\end{tabular}




\subsection{MPDL3280A}

MPDL3280A (Roche) is an engineered anti-PD-L1 antibody. In the initial Phase I dose-escalation study involving 171 patients with locally advanced or metastatic tumors, no dose-limiting toxicities were observed and the maximum tolerated dose was not identified (Herbst et al., 2013). Overall it was observed that patients with PD-L1 positive tumors (from archival samples) had a higher response rate as compared to PD-L1 negative tumors. It demonstrated a promising overall response rate in patients with metastatic bladder cancer whose tumors were characterized as PD-L1 positive (Powles et al., 2014). The overall response rate was $52 \%$ in patients with previously treated metastatic PD-L1 positive bladder cancer as compared to $11 \%$ in patients who were PD-L1 negative. The median time to response was 42 days and complete response was observed in $7 \%$ of PD-L1 positive patients. In view of these spectacular results, the FDA granted MPDL3280A a breakthrough therapy designation in bladder cancer. Of note, it is for the first time that an agent has received this designation in bladder cancer.

Currently, studies with MPDL3280A are being designed to test for PD-L1 expression on tumor cells by immunohistochemistry (IHC) and analyze efficacy according to different IHC strata (ClinicalTrials.gov identifier: NCT01846416). A Phase II study of MPDL3280A is currently evaluating its role in patients with metastatic urothelial bladder cancer ("A Study of MPDL3280A in Patients With Locally Advanced or Metastatic Urothelial Bladder Cancer,"). This study will generate additional data in patients who are ineligible for platinum-containing therapy (treatment-naive) and those who have progressed following a prior platinum-based chemotherapy.

\subsection{MEDI4736}

MEDI4736 (AstraZeneca) is a human monoclonal antibody directed against PD-L1. An encouraging clinical activity was seen in a Phase I clinical trial of 26 patients with advanced solid tumors (Lutzky et al., 2014). With a manageable toxicity profile, four confirmed partial responses and a disease control rate of $46 \%$ was observed. Again no DLTs or maximum tolerated dose were identified. Further development of this agent is underway with expansion cohorts for bladder cancer and multiple other tumor types. The preliminary safety and durable clinical efficacy profile of MEDI4736 has also led to initiation of a Phase III study in non-small cell lung cancer ("A Global Study to Assess the Effects of MEDI4736 Following Concurrent Chemoradiation in Patients With Stage III Unresectable Non-Small Cell Lung Cancer (PACIFIC)"). Studies are currently ongoing both as a monotherapy and combination in several tumor types ("Phase 1 Safety and Tolerability of MEDI4736 in Combination With Dabrafenib and Trametinib or With Trametinib Alone" ; "Phase II Study of MEDI4736 Monotherapy in the Treatment of Patients With Recurrent or Metastatic SCCHN").

\subsection{Nivolumab}

Nivolumab (Bristol-Myers Squibb) is another agent of great interest and a fully human IgG4 monoclonal antibody against PD-1. A Phase I/II trial of nivolumab in combination with ipilimumab is currently enroling patients with bladder cancer ("A Phase 1/2, Open-label Study of Nivolumab Monotherapy or Nivolumab Combined With Ipilimumab in Subjects With Advanced or Metastatic Solid Tumors,"). Future role of this agent in bladder cancer will largely depend on the results of this study. The first-in-human Phase I study previously evaluated its safety and tolerability in 39 patients with advanced refractory solid tumors (Brahmer et al., 2010). This agent was well tolerated to the maximum planned dose of $10 \mathrm{mg} / \mathrm{kg}$; one durable complete response and two partial responses were observed. Subsequently, a large Phase I study in 207 patients also confirmed objective responses in patients with melanoma, non-small cell lung, and renal cancers (Brahmer et al., 2012). Prolonged stabilization of disease at 24 weeks was observed in upto $41 \%$ patients. Nivolumab is currently in advanced stages of development in patients with malignant melanoma and renal cancer ("Study of Nivolumab vs. Everolimus in Pre-Treated Advanced or Metastatic Clear-cell Renal Cell Carcinoma,").

\subsection{Lambrolizumab}

Lambrolizumab (Merck), also formerly known as MK-3475, is a humanized monoclonal IgG4 antibody that acts against PD-1. Its role in advanced bladder cancer is currently being evaluated ("Study of MK-3475 in Participants With Advanced Solid Tumors", 2014). Previously it received a breakthrough therapy designation from the FDA after promising results were seen in patients with advanced melanoma. MK-3475 is currently being studied in advanced triple negative breast, head and neck, and urothelial cancers ("Study of MK-3475 in Participants With Advanced Solid Tumors", 2014).

\section{Conclusions}

Because treatment with immunotherapy agents has lead to clinical responses in various cancers, it seems that immuno-oncology will dominate the oncology enterprise for the rest of this decade. Above discussed results 
support further development of these agents for the treatment of patients with advanced bladder cancer. However, a predictive biomarker that can be used to select patients for treatment with these agents has not yet been identified. Expression of PD-L1 on immune cells or tumor cells may be potentially helpful, because a subset of patients who were deemed to be PD-L1 positive were found to have clinical responses with MPDL3280A (Powles et al., 2014). The relationship between PD-L1 status and efficacy in patients with urothelial cancer warrants further exploration. Future high quality biomarker-driven clinical trials are essential for spearheading this new approach.

\section{Funding}

None.

\section{Conflict of Interest}

None.

\section{References}

A Global Study to Assess the Effects of MEDI4736 Following Concurrent Chemoradiation in Patients With Stage III Unresectable Non-Small Cell Lung Cancer (PACIFIC). Retrieved August, 2014, from https://clinicaltrials.gov/ct2/show/NCT02125461?term=MEDI4736\&rank=7

A Phase 1/2, Open-label Study of Nivolumab Monotherapy or Nivolumab Combined With Ipilimumab in Subjects With Advanced or Metastatic Solid Tumors. from http://clinicaltrials.gov/show/NCT01928394

A Study of MPDL3280A in Patients With Locally Advanced or Metastatic Urothelial Bladder Cancer. Retrieved August, 2014, from http://www.clinicaltrials.gov/ct2/show/NCT02108652?term=MPDL3280A\&rank=4

Babjuk, M., Oosterlinck, W., Sylvester, R., Kaasinen, E., Bohle, A., \& Palou-Redorta, J. (2008). EAU guidelines on non-muscle-invasive urothelial carcinoma of the bladder. European Urology, 54(2), 303-314. http://dx.doi.org/10.1016/j.eururo.2008.04.051

Bellmunt, J., Theodore, C., Demkov, T., Komyakov, B., Sengelov, L., Daugaard, G., . . . von der Maase, H. (2009). Phase III trial of vinflunine plus best supportive care compared with best supportive care alone after a platinum-containing regimen in patients with advanced transitional cell carcinoma of the urothelial tract. Journal of Clinical Oncology, 27(27), 4454-4461. http://dx.doi.org/10.1200/jco.2008.20.5534

Blank, C., \& Mackensen, A. (2007). Contribution of the PD-L1/PD-1 pathway to T-cell exhaustion: an update on implications for chronic infections and tumor evasion. Cancer Immunology, Immunotherapy, 56(5), 739-745. http://dx.doi.org/10.1007/s00262-006-0272-1

Brahmer, J. R., Drake, C. G., Wollner, I., Powderly, J. D., Picus, J., Sharfman, W. H., . . Topalian, S. L. (2010). Phase I study of single-agent anti-programmed death-1 (MDX-1106) in refractory solid tumors: safety, clinical activity, pharmacodynamics, and immunologic correlates. Journal of Clinical Oncology, 28(19), 3167-3175. http://dx.doi.org/10.1200/jco.2009.26.7609

Brahmer, J. R., Tykodi, S. S., Chow, L. Q., Hwu, W. J., Topalian, S. L., Hwu, P., . . Wigginton, J. M. (2012). Safety and activity of anti-PD-L1 antibody in patients with advanced cancer. New England Journal of Medicine, 366(26), 2455-2465. http://dx.doi.org/10.1056/NEJMoa1200694

Butte, M. J., Keir, M. E., Phamduy, T. B., Sharpe, A. H., \& Freeman, G. J. (2007). Programmed death-1 ligand 1 interacts specifically with the B7-1 costimulatory molecule to inhibit T cell responses. Immunity, 27(1), 111-122. http://dx.doi.org/10.1016/j.immuni.2007.05.016

Carthon, B. C., Wolchok, J. D., Yuan, J., Kamat, A., Ng Tang, D. S., Sun, J., . . Sharma, P. (2010). Preoperative CTLA-4 blockade: tolerability and immune monitoring in the setting of a presurgical clinical trial. Clinical Cancer Research, 16(10), 2861-2871. http://dx.doi.org/10.1158/1078-0432.ccr-10-0569

Chen, D. S., Irving, B. A., \& Hodi, F. S. (2012). Molecular pathways: next-generation immunotherapy--inhibiting programmed death-ligand 1 and programmed death-1. Clinical Cancer Research, 18(24), 6580-6587. http://dx.doi.org/10.1158/1078-0432.ccr-12-1362

Chen, L., \& Flies, D. B. (2013). Molecular mechanisms of T cell co-stimulation and co-inhibition. Nature Reviews: Immunology, 13(4), 227-242. http://dx.doi.org/10.1038/nri3405

Elsasser-Beile, U., Gutzeit, O., Bauer, S., Katzenwadel, A., Schultze-Seemann, W., \& Wetterauer, U. (2000). Systemic and local immunomodulatory effects of intravesical BCG therapy in patients with superficial urinary bladder carcinomas. Journal of Urology, 163(1), 296-299. 
Hall, M. C., Chang, S. S., Dalbagni, G., Pruthi, R. S., Seigne, J. D., Skinner, E. C., . . Schellhammer, P. F. (2007). Guideline for the management of nonmuscle invasive bladder cancer (stages Ta, T1, and Tis): 2007 update. Journal of Urology, 178(6), 2314-2330. http://dx.doi.org/10.1016/j.juro.2007.09.003

Herbst, R., Gordon, M., Fine, G., Sosman, J., Soria, J., Hamid, O., . . Hodi, S. (2013). A study of MPDL3280A, an engineered PD-L1 antibody in patients with locally advanced or metastatic tumors. J Clin Oncol. 2013;31(suppl; abstr 3000).

Herr, H. W. (1997). Tumour progression and survival in patients with T1G3 bladder tumours: 15-year outcome. British Journal of Urology, 80(5), 762-765.

Hodi, F. S., O'Day, S. J., McDermott, D. F., Weber, R. W., Sosman, J. A., Haanen, J. B., . . Urba, W. J. (2010). Improved survival with ipilimumab in patients with metastatic melanoma. New England Journal of Medicine, 363(8), 711-723. http://dx.doi.org/10.1056/NEJMoa1003466

Iwai, Y., Ishida, M., Tanaka, Y., Okazaki, T., Honjo, T., \& Minato, N. (2002). Involvement of PD-L1 on tumor cells in the escape from host immune system and tumor immunotherapy by PD-L1 blockade. Proceedings of the National Academy of Sciences of the United States of America, 99(19), 12293-12297. http://dx.doi.org/10.1073/pnas.192461099

Loehrer, P. J., Sr., Einhorn, L. H., Elson, P. J., Crawford, E. D., Kuebler, P., Tannock, I., . . . et al. (1992). A randomized comparison of cisplatin alone or in combination with methotrexate, vinblastine, and doxorubicin in patients with metastatic urothelial carcinoma: a cooperative group study. Journal of Clinical Oncology, 10(7), 1066-1073.

Lutzky, J., Antonia, S., Haskins, A., Li, X., Robbins, P., Shalabi, A., . . Segal, N. (2014). A phase 1 study of MEDI4736, an anti-PD-L1 antibody, in patients with advanced solid tumors. Journal of Clinical Oncology, 32:5s, (suppl; abstr 3001).

Mitropoulos, D. N. (2005). Novel insights into the mechanism of action of intravesical immunomodulators. In Vivo, 19(3), 611-621.

Morales, A., Eidinger, D., \& Bruce, A. W. (1976). Intracavitary Bacillus Calmette-Guerin in the treatment of superficial bladder tumors. Journal of Urology, 116(2), 180-183.

Mu, C. Y., Huang, J. A., Chen, Y., Chen, C., \& Zhang, X. G. (2011). High expression of PD-L1 in lung cancer may contribute to poor prognosis and tumor cells immune escape through suppressing tumor infiltrating dendritic cells maturation. Med Oncol, 28(3), 682-688. http://dx.doi.org/10.1007/s12032-010-9515-2

Peggs, K. S., Quezada, S. A., Korman, A. J., \& Allison, J. P. (2006). Principles and use of anti-CTLA4 antibody in human cancer immunotherapy. Current Opinion in Immunology, 18(2), 206-213. http://dx.doi.org/10.1016/j.coi.2006.01.011

Phase 1 Safety and Tolerability of MEDI4736 in Combination With Dabrafenib and Trametinib or With Trametinib Alone. Retrieved August, 2014, from https://clinicaltrials.gov/ct2/show/NCT02027961?term=MEDI4736\&rank=1

Phase II Study of MEDI4736 Monotherapy in the Treatment of Patients With Recurrent or Metastatic SCCHN. Retrieved August, 2014, from https://clinicaltrials.gov/ct2/show/NCT02207530?term=MEDI4736\&rank=10

Phase II Trial of Gemcitabine, Cisplatin, Plus Ipilimumab as First-line Treatment for Patients With Metastatic Urothelial Carcinoma: Hoosier Oncology Group GU10-148. Retrieved August, 2014, from http://www.clinicaltrials.gov/ct2/show/NCT01524991?term=ipilimumab+urothelial\&rank=1

Powles, T., Vogelzang, N., Fine, G., Eder, J., Fadi Braiteh, Loriot, Y., . . Petrylak, D. (2014). Inhibition of PD-L1 by MPDL3280A and clinical activity in pts with metastatic urothelial bladder cancer (UBC). Journal of Clinical Oncology, 32:5s, 2014 (suppl; abstr 5011).

Raghavan, D. (2003). Chemotherapy and cystectomy for invasive transitional cell carcinoma of bladder. Urologic Oncology, 21(6), 468-474.

Rajala, P., Kaasinen, E., Rintala, E., Jauhiainen, K., Nurmi, M., Alfthan, O., \& Lahde, M. (1992). Cytostatic effect of different strains of Bacillus Calmette-Guerin on human bladder cancer cells in vitro alone and in combination with mitomycin C and interferon-alpha. Urological Research, 20(3), 215-217.

Ratliff, T. L., Gillen, D., \& Catalona, W. J. (1987). Requirement of a thymus dependent immune response for BCG-mediated antitumor activity. Journal of Urology, 137(1), 155-158. 
SEER Stat Fact Sheets: Bladder Cancer. (2014). Retrieved July, 2014, from http://seer.cancer.gov/statfacts/html/urinb.html

Shelley, M. D., Court, J. B., Kynaston, H., Wilt, T. J., Fish, R. G., \& Mason, M. (2000). Intravesical Bacillus Calmette-Guerin in Ta and T1 Bladder Cancer. Cochrane Database Syst Rev(4), CD001986. http://dx.doi.org/10.1002/14651858.cd001986

Strome, S. E., Dong, H., Tamura, H., Voss, S. G., Flies, D. B., Tamada, K., . . Chen, L. (2003). B7-H1 blockade augments adoptive T-cell immunotherapy for squamous cell carcinoma. Cancer Research, 63(19), 6501-6505.

Study of MK-3475 in Participants With Advanced Solid Tumors (2014). Retrieved August, 2014, from http://www.clinicaltrials.gov/ct2/show/NCT01848834?term=mk-3475\&rank=3

Study of Nivolumab vs. Everolimus in Pre-Treated Advanced or Metastatic Clear-cell Renal Cell Carcinoma. Retrieved

August, 2014, http://www.clinicaltrials.gov/ct2/show/NCT01668784?term=Nivolumab\&rank=20

Sylvester, R. J., van der Meijden, A. P., Witjes, J. A., \& Kurth, K. (2005). Bacillus calmette-guerin versus chemotherapy for the intravesical treatment of patients with carcinoma in situ of the bladder: a meta-analysis of the published results of randomized clinical trials. Journal of Urology, 174(1), 86-91; discussion 91-82. http://dx.doi.org/10.1097/01.ju.0000162059.64886.1c

Taniguchi, K., Koga, S., Nishikido, M., Yamashita, S., Sakuragi, T., Kanetake, H., \& Saito, Y. (1999). Systemic immune response after intravesical instillation of bacille Calmette-Guerin (BCG) for superficial bladder cancer. Clinical and Experimental Immunology, 115(1), 131-135.

Witjes, J. A. (2006). Management of BCG failures in superficial bladder cancer: a review. European Urology, 49(5), 790-797. http://dx.doi.org/10.1016/j.eururo.2006.01.017

Yang, J., Riella, L. V., Chock, S., Liu, T., Zhao, X., Yuan, X., . . Sayegh, M. H. (2011). The novel costimulatory programmed death ligand 1/B7.1 pathway is functional in inhibiting alloimmune responses in vivo. Journal of Immunology, 187(3), 1113-1119. http://dx.doi.org/10.4049/jimmunol.1100056

Zlotta, A. R., Fleshner, N. E., \& Jewett, M. A. (2009). The management of BCG failure in non-muscle-invasive bladder cancer: an update. Can Urol Assoc J, 3(6 Suppl 4), S199-205.

\section{Copyrights}

Copyright for this article is retained by the author(s), with first publication rights granted to the journal.

This is an open-access article distributed under the terms and conditions of the Creative Commons Attribution license (http://creativecommons.org/licenses/by/3.0/). 